\title{
203 - Decisional capacity and advance care planning in older people who are incarcerated Dionne Hart, M.D.
}

Abstract:

There is a growing number of older people incarcerated across the United States. With a population of greater than 300 million, the US has $5 \%$ of the world's population, yet incarcerates $25 \%$ of the world's prisoners. From 2000 to 2005, the percentage of prisoners in federal and state correctional institutions who were 55 and older increased by $33 \%$. According to the American Civil Liberties Union older prison population has climbed $1300 \%$ since the 1980 s, with 125,000 inmates aged 55 or older incarcerated.

Correctional facilities are the largest mental health institutions with 1 out of 5 individuals with serious mental health or substance use disorders. These facilities lack the capacity to provide long-term care for those with severe physical or mental health disorders even in the most ideal circumstances. Individuals within the criminal justice system have a higher burden of chronic physical and health disorders and have a lower life expectancy.

Health care decision making is one area where patients in custody have autonomy in discussing advance directives, substitute decision makers and medical decision making. However, prisoners are at risk of suboptimal care, unmet palliative and end of life care needs, and lacking or inappropriate surrogates. Without documentation of advance directives or surrogates there are bureaucratic, practical, and legal barriers particularly for those without family or friends. In addition, some individuals involved in the correctional system's only surviving family members may also be their victims, thus have a conflict of interest.

This presentation will explore capacity and substitute decision making for individuals involved in the criminal justice system who have severe mental and physical health disorders. A case description will be used to illustrate a decision-making tree for patients who are incarcerated. As the world population continues to age, the number of older people who are incarcerated and unable to make healthcare decisions will continue to increase. In this special population, correctional system clinicians and providers need to be familiar with strategies to address the need for advance care planning before older people lose decisional capacity.

\section{4 - Psychological distress and support needs of community residing older adults in urban India - An exploratory study}

Authors: Jayashree Dasgupta, Meenakshi Chopra

Affiliation: Samvedna Senior Care, Delhi, India

Background: COVID pandemic in India, lockdowns and an unprepared health system has affected wellbeing of older adults. Low public awareness about mental health issues and stigma also contribute to low help seeking. Exploring impact of COVID on mental health of older adults and understanding support needs is essential.

Research Objective: To examine mental wellbeing and coping strategies used by urban community residing older adults during the pandemic in India.

Method: As part of an ongoing community engagement initiative with older adults and their families, an online survey was conducted during the first wave of the pandemic in April/May 2020. Sociodemographic details and information on coping strategies were gathered. The five-item General 